\title{
Improved diagnostic accuracy in differentiating malignant and benign lesions using single-voxel proton MRS of the breast at $3 \mathrm{~T}$ MRI
}

\begin{abstract}
AIM: To investigate the diagnostic accuracy of single-voxel proton magnetic resonance spectroscopy (SV (1)H MRS) by quantifying total choline-containing compounds (tCho) in differentiating malignant from benign lesions, and subsequently, to analyse the relationship of tCho levels in malignant breast lesions with their histopathological subtypes. MATERIALS AND METHODS: A prospective study of SV $1 \mathrm{H}$ MRS was performed following dynamic contrast-enhanced magnetic resonance imaging (DCE-MRI) in 61 women using a 3 T MR system. All lesions $(n=57)$ were analysed for characteristics of morphology, contrast-enhancement kinetics, and tCho peak heights at SV (1)H MRS that were two-times above baseline. Subsequently, the tCho in selected lesions $(n=32)$ was quantified by calculating the area under the curve, and a tCho concentration equal to or greater than the cutoff value was considered to represent malignancy. The relationship between tCho in invasive ductal carcinomas (IDCs) and their Bloom \& Richardson grading of malignancy was assessed. RESULTS: Fifty-two patients (57 lesions; 42 malignant and 15 benign) were analysed. The sensitivity, specificity, positive predictive value (PPV), and negative predictive value (NPV), of predicting malignancy were 100, 73.3, 91.3, and 100\%, respectively, using DCE-MRI and 95.2, 93.3, 97.6, and 87.5\%, respectively, using SV (1)H MRS. The tCho cut-off for receiver operating characteristic (ROC) curve was $0.33 \mathrm{mmol} / \mathrm{l}$. The relationship between tCho levels in malignant breast lesions with their histopathological subtypes was not statistically significant ( $\mathrm{p}=0.3$ ). CONCLUSION: Good correlation between tCho peaks and malignancy, enables SV (1)H MRS to be used as a clinically applicable, simple, yet non-invasive tool for improved specificity and diagnostic accuracy in detecting breast cancer.
\end{abstract}

Keyword: Breast cancer; Malignant; Benign lesions; Single-voxel proton magnetic resonance spectroscopy; Breast; Magnetic resonance imaging 